\title{
Fermentador inteligente con tecnología de fermentación controlada para estandarizar procesos de fermentación de cafés de especialidad
}

\section{Smart fermenter with controlled fermentation technology to standardize specialty coffee fermentation processes}

\author{
Carbajal-Guerreros, Ismael [(D) 0000-0003-3881-8572]I; Pilco-Valles, Herberth [(D) 0000-0002-7768-2359];; \\ García-Herrera, Flor Aracely [(D) 0000-0003-2090-3146] ; Coronel-Rufasto, Ivan [(1) 0000-0002-4010-0239]; \\ Gonzales-Diaz, Jose Rolando [(1D) 0000-0003-0277-5742]'; Cabanillas-Pardo, Lenin [(D) 0000-0002-1059-8064]।
}

'Prolnnóvate, Perú

$\triangle$ gerenciafrutosdeselva@gmail.com

Recibido: 10/12/2021; $\quad$ Aceptado: 10/01/2022; $\quad$ Publicado: 20/01/2022

Resumen: El cultivo del café como materia prima es muy apreciada por la bebida resultante de agradabable sabor y valor socioeconómico. Nuestro objetivo fue diseñar un dispostivo fermentador inteligente con tecnología de fermentación controlada para estandarizar procesos de fermentación de cafés de especialidad. Para ello realizamos un estudio con diseño descriptivo comparativo con enfoque basado en desarrollo tecnológico que dividimos en cuatro etapas de contrucción y para validarlo se utilizaron 150 kilos batch de grado de café despulpado fresco. Logramos construir un dispositivo fermentador inteligente con tecnología de fermentación controlada para estandarizar procesos de fermentación de cafés de especialidad con el que se aplica un protocolo de funcionamiento de prototipo eficiente, con la capacidad de estandarizar procesos de fermentación controlada para cafés de especilidad. El prototipo construido permite a través de un protocolo fermentar de forma controlada y eficiente el café a partir del cual obtuvimos 84 puntos SCAA de valoración y el control de la temperatura logrado ayuda a equilibrar los procesos bioquímicos que se ve reflejado en las puntuaciones de tasas con características de sabores, fragancias uniformes y perfiles suaves que le otorgan al café 84 puntos.

Palabras clave: café; fermentación; medición; pergamino; taza; procesos

\begin{abstract}
The cultivation of coffee as a raw material is highly appreciated for the resulting beverage with a pleasant taste and socioeconomic value. Our objective was to design an intelligent fermentation device with controlled fermentation technology to standardize fermentation processes for specialty coffees. For this, we carried out a study with a comparative descriptive design with an approach based on technological development that we divided into four construction stages and to validate it, 150 batch kilos of fresh pulped coffee grade were used. We managed to build an intelligent fermentation device with controlled fermentation technology to standardize specialty coffee fermentation processes with which an efficient prototype operating protocol is applied, with the ability to standardize controlled fermentation processes for specialty coffees. The built prototype allows, through a protocol, to ferment the coffee in a controlled and efficient way, from which we obtained 84 SCAA assessment points and the temperature control achieved helps to balance the biochemical processes that is reflected in the rate scores with characteristics of flavors, uniform fragrances and smooth profiles that give the coffee 84 points.
\end{abstract}

Keywords: bowl; coffee; fermentation; measurement; parchment; processes

Cómo citar / Citation: Carbajal-Guerreros, I., Pilco-Valles, H., García-Herrera, F. A., Coronel-Rufasto, I., GonzalesDiaz, J. R. \& Cabanillas-Pardo, L. (2022). Fermentador inteligente con tecnología de fermentación controlada para estandarizar los procesos de fermentación de cafés de especialidad. Revista agrotecnológica amazónica, 2(I), e303. https://doi.org//0.51252/raa.v2il.303 


\section{Introducción}

Dentro del campo agrícola el café es uno de los cultivos de mayor importancia y ha demostrado tener peso socioeconómico a lo largo de la historia (Al-Abdulkader et al., 2018). Asimismo, en muchos países es un producto de alto consumo y grandes extensiones de plantación (Sayed et al., 2019). Su consumo llega a tener una tasa de crecimiento media anual del 3,5\% que va en aumento (Magalhães Júnior et al., 202I). En este sentido, (Smith Dumont et al., 2019) consideran pertinente el conocimiento técnico-agrícola para su productividad y sostenibilidad; caso contrario, el rendimiento del cultivo y futura comercialización se verán afectados.

La producción y comercialización del café (Coffea arabica) representa el sustento de millones de agricultores (Pham et al., 2019), siendo un producto básico e importante en el mundo (Lopes et al., 2020) por brindar beneficios a la salud humana (Bonilla Medina, 2017). De acuerdo al análisis estadístico realizado por (Ocampo Lopez \& Alvarez-Herrera, 2017) sobre las cafeteras reportadas por la Organización Internacional del Café, países como Brasil, Vietnam, Indonesia, Honduras, Nicaragua y Perú, son tendencias de surgimiento en su producción.

En el Perú, el café es uno de los principales productos de agroexportación, contando con 420 mil ha dedicadas al cultivo, además la producción representa el sustento de 223 mil familias (Julca-Otiniano et al., 2018). Asimismo, Perú es un referente a nivel mundial de cafés especiales, ocupando el 2do lugar a nivel mundial como productor/exportador de café orgánico, siendo el principal proveedor de café de Estados Unidos con el sello de Fair Trade (Estevez et al., 2017). Pese a las dificultades logísticas del proceso de cosecha y embarque incitados por la pandemia de la covid-19, la producción y exportación de café sumaron un total de 4739000 quintales, por un valor de 658 millones de dólares, lo que evidencia su importancia en el crecimiento económico de una nación (Junta Nacional del Café, 202I).

En la región San Martín, el Valle del Alto Mayo es considerado una de las principales zonas productora de café, pues su principal actividad socio-económica es la caficultura, teniendo a más de 35 mil familias dedicadas a la producción (Rojas-Ruiz et al., 2020). Por tal motivo, la mayoría de empresas del rubro se encuentran ubicadas en esta parte de la región, siendo las organizaciones y cooperativas formadas por pequeños productores las que ofertan cafés especiales certificados de alta calidad.

La empresa, Frutos de selva, es una de las empresas ubicadas en el Valle del Alto Mayo que tiene como actividad principal la comercialización de grano de café logrando vender el año 2016 la cantidad de 12 contenedores de grano de café verde, el $80 \%$ al mercado nacional y el $20 \%$ al mercado internacional, además, la empresa aumento sus alianzas institucionales y comerciales para incrementar su capacidad exportable de grano de café, junto a esto la Cooperativa ha desarrollado proyectos productivos con apoyo de entes del estado, logrando con ello mejorar la productividad y calidad del producto, haciéndolo más competitivo en el ámbito comercial, además de implementar con tecnología eficaz para mejorar los procesos.

Por otra parte, Frutos de selva tiene un manejo post cosecha donde el proceso de fermentación se realiza de forma convencional y artesanal, se cuenta con un protocolo, sin embargo, no es posible el control de tiempo, humedad, temperatura y $\mathrm{pH}$, por lo que no es posible conocer volúmenes de masa concentrado por tipo de cajón, variedad y pisos actitudinales. Al no tener el control de las citadas variables, el proceso de fermentación es bastante heterogéneo, razón por lo cual al solo se llega a obtener en el mejor de los casos 82.5 SCAA de puntuación de calidad, pues, las características del grano no se desarrollan, perdiendo 
su valor para el mercado de cafés especiales, pasando el $97 \%$ a ser convencionales y por ende su valor de precio decrece.

La post cosecha es una actividad muy importante para la obtención del producto final, la misma que el productor en un $80 \%$ no lo lleva a cabo aplicando criterios técnicos que ayuden a que este proceso sea llevado de manera eficiente, siendo la fermentación un cuello de botella, ya que el productor no tiene conocimiento del tiempo optimo, temperatura y otros factores importantes, y determinantes en el proceso, razón por la cual lo llevan de acuerdo a su criterio no garantizando la calidad del producto final.

Por tal motivo, es urgente mejorar los procedimientos de manejo de post cosecha, especialmente el proceso de fermentación, que es donde el grano de café mediante cambios biológicos y químicos adquiere los atributos deseables que lo convierte en un café superior. Estos tipos de café son vendidos a tostadores de EE.UU y Europa a precios superiores a los $\$ 200.00$ dólares americanos por quintal de $46 \mathrm{~kg}$. La Cooperativa busca incrementar el volumen de oferta de estos tipos de cafés, ya que no satisface la demanda de sus clientes.

Por lo descrito, el objetivo del proyecto es diseñar y construir prototipo fermentador inteligente con tecnología de fermentación controlada que permita estandarizar los procesos de fermentación para la obtención de cafés de especialidad, pues para la estandarización de los procesos de fermentación es necesario controlar y manejar de manera eficiente el tiempo, temperatura, ph, al momento del proceso, el cual tiene que ser evaluado en diferentes tiempos, hasta lograr encontrar el perfil ideal del grano en estudio.

\section{Materiales y métodos}

\section{Unidad de Análisis}

El diseño es descriptivo comparativo; por que busca caracterizar un fenómeno o hecho en base a la información recogida de varias muestras, en base a la comparación de los resultados encontrados en las mismas como también recoge información de una situación sin ejercer un control de un tratamiento. La población es el prototipo fermentador con 150 kilos batch de grano de café despulpado fresco, el cual se acopiará durante la ejecución del proyecto. El prototipo fermentador construido funcional es el $p$.

Frutos de selva contó con infraestructura para el acopio de grano de café, la misma que estuvo implementada por un laboratorio de control de calidad con los equipos básicos para realizar el análisis físico sensorial de grano de café, asimismo se encuentro comercializando granos de café convencionales al mercado nacional y especiales al mercado internacional, este último con oferta creciente cada año. Asimismo, con la aparición de nuevos segmentos de mercados la demanda de este tipo de café fue cada vez más, incrementando la producción de cafés especiales de la empresa en un $10 \%$, mediante el cual pretendió abarcar más el segmento de cafés especiales, ya que se contó con clientes potenciales para estos tipos de café.

\section{Plan metodológico del proyecto}

La ejecución del proyecto estuvo dada por etapas (pasos críticos), donde en cada uno de ellos se realizó actividades direccionadas a cumplir los objetivos del proyecto, y se desarrolló de la siguiente manera: 
Primera etapa: Se realizó el diseño técnico del prototipo con aporte de experiencias en campo y un especialista que brindó asesoramiento para diseño y elaboración de los planos de dos modelos de prototipos con capacidad de estandarizar los procesos de fermentación basados en temperatura, ph, volumen de masa, tiempos de proceso. Se desarrolló la evaluación de la estructura física de acuerdo a los parámetros de uso, y por último la simulación del prototipo y su potencial funcionamiento de todas sus partes.

Segunda etapa: Desarrollo y funcionamiento de uno de los dos prototipos funcionales, se identificó las zonas con potencial para la recolección de muestras, que fueron evaluadas para su viabilidad y uso con el prototipo. Primeras pruebas de funcionamiento del prototipo, las muestras evaluadas fueron analizadas en laboratorio tanto físico como organoléptico.

Tercera etapa: Establecimiento de los parámetros óptimos de funcionamiento y validación, múltiples evaluaciones, hasta estandarizar el proceso; las muestras evaluadas analizadas en laboratorio para determinar la calidad físico sensorial del grano, con los resultados eficientes finales se elaboró un protocolo de funcionamiento de prototipo para su aplicación continua.

Cuarta etapa: Gestión y cierre del proyecto.

\section{Flujograma del proceso de desarrollo del prototipo fermentador Clasificación del café cosechado}

Figura I. Flujograma del proceso de desarrollo del prototipo fermentador inteligente

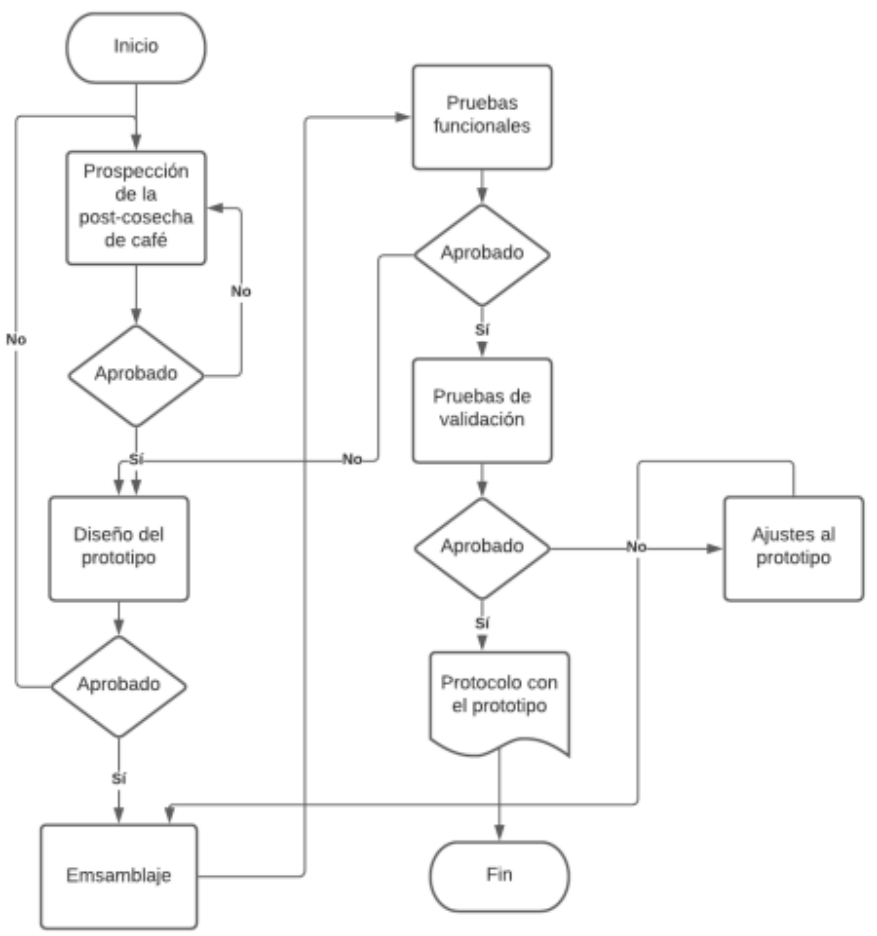

\section{Criterios de las variables vinculantes al prototipo}

Utilizamos el beneficio semi húmedo - Honey, los frutos maduros son despulpados y secados con el mucílago adherido al grano (Rodriguez et al., 2020). Utilizamos el prototipo construido, esparciendo los granos en capas delgadas de $3 \mathrm{~cm}$ y removiendo cada 20 minutos a temperaturas 
máximas de $35{ }^{\circ} \mathrm{C}$ y mínimas de $20{ }^{\circ} \mathrm{C}$ controladas con colectores de aire a base de energía solar evitando mezclar granos con diferente contenido de humedad. Se realizó monitoreo con el software del prototipo (da Costa et al., 202I). La clasificación antes del despulpado y el secado son las etapas importantes en la calidad final para que presente atributos sensoriales dulces $y$ frutales, conservando el nivel de acidez para ser comercialmente bien valorado. Las muestras humedad entre $12 \%$ a $14 \%$ se dejaron en reposo en parihuelas de madera separadas de la pared, con una altura entre 10 y $15 \mathrm{~cm}$ del suelo durante una semana en una bodega seca y ventilada, con temperatura menor a $20^{\circ} \mathrm{C}$ y humedad relativa del $65 \%$ al $70 \%$ para evitar rehumedecimiento del producto (Sotelo-Valer et al., 2020). El almacén de café estuvo separado del centro de beneficio y cerca de la bodega se evitaron olores.

De la experiencia del equipo técnico, productores y experto consultor se determinó que se debe tener la siguientes variables o criterios para el diseño del prototipo.

Tabla I. Criterios de las variables vinculantes al prototipo

\begin{tabular}{|c|c|}
\hline Requisitos & Propuesta \\
\hline $\begin{array}{c}\text { Debe contener los granos de café en baba durante la } \\
\text { fermentación y drenar el mucilago en las horas de } \\
\text { fermentación }\end{array}$ & $\begin{array}{l}\text { A través de agujeros incorporados en el } \\
\text { prototipo fermentador. }\end{array}$ \\
\hline Debe permitir el drenado del mucílago. & Por gravedad y presión. \\
\hline $\begin{array}{l}\text { Debe permitir remover los granos de café durante la } \\
\text { fermentación sin causar daños. }\end{array}$ & $\begin{array}{l}\text { A través de un mecanismo } \\
\text { automatizado, paletas o tornillo sin fin. }\end{array}$ \\
\hline $\begin{array}{c}\text { Deber permitir la aireación de los granos de café } \\
\text { durante la fermentación. }\end{array}$ & $\begin{array}{l}\text { A través de ranuras que permitan cerrar } \\
\text { y abrir tipo agujeros. }\end{array}$ \\
\hline $\begin{array}{l}\text { Debe mantener la temperatura durante la } \\
\text { fermentación. }\end{array}$ & $\begin{array}{c}\text { Cajón fermentador cerrado } \\
\text { enchaquetado con un material aislante } \\
\text { de calor. }\end{array}$ \\
\hline $\begin{array}{c}\text { Debe permitir la homogenización de la masa de café } \\
\text { fermentante. }\end{array}$ & $\begin{array}{l}\text { Giro automatizado del cajón } \\
\text { fermentador. }\end{array}$ \\
\hline $\begin{array}{l}\text { Debe permitir la medición de la temperatura y } \mathrm{pH} \\
\text { de la masa fermentante. }\end{array}$ & $\begin{array}{l}\text { Sensores de temperatura y } \mathrm{pH} \\
\text { instalador en el cajón fermentador. }\end{array}$ \\
\hline
\end{tabular}

\section{Resultados y discusión}

\section{Flujograma del proceso de fermentación que realiza el fermentador diseñado}

Este subproceso se desarrolló dentro del proceso de postcosecha de café, el cual se tuvo en cuenta para la validación. Acá se trata la fermentación. Presentamos el siguiente diagrama de flujo (Figura 2) para esquematizar y partir con los diseños del prototipo. 
Figura 2. Flujograma del proceso de fermentación que realiza el fermentador diseñado.

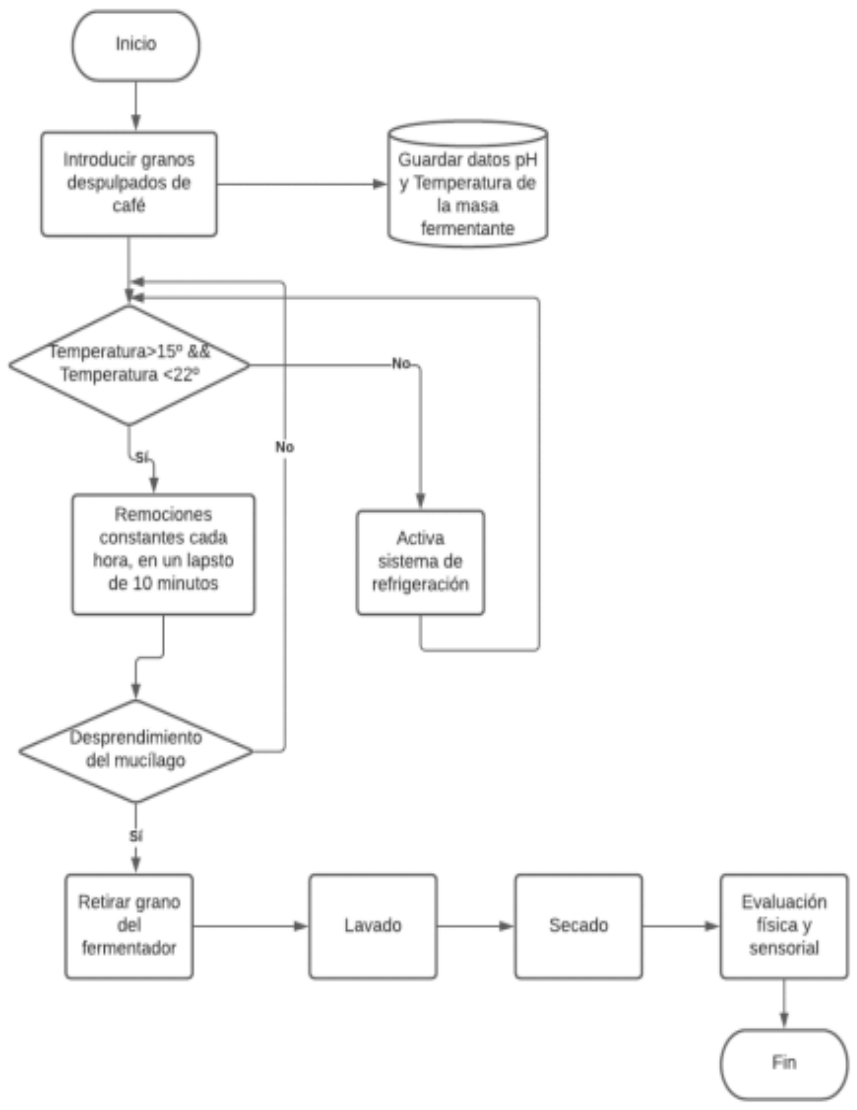

Como se observa el en siguiente diagrama flujo, el fermentador se basará su control en la temperatura. Con la base de esto se inició a diseñó la propuesta, del cual hemos codificado y se definió un fermentador adecuado con las expectativas descritas, el fermentador fue diseñado para una capacidad de I50 kilos de grano despulpado para fermentarse.

\section{Prototipo}

Los resultados obtenidos se elaboraron en base a los requerimientos determinados:

- Fermentador cilindro, con chaqueta de agua (aislante de factores externos, sistema de aireación, motor rotatorio de palancas tipo hélices dentro de la masa).

- Sistema de sensores conectados a la masa (Ph, Ox y Temperatura) para controlar los sistemas del motor, y con los datos determinar el algoritmo inteligente para afinar el proceso de fermentación controlada.

- Sistema conecta a ductos de agua, para facilitar el lavado del grano.

- Sistema de salida de mucílago y del grano. 
Figura 3. Planos del prototipo

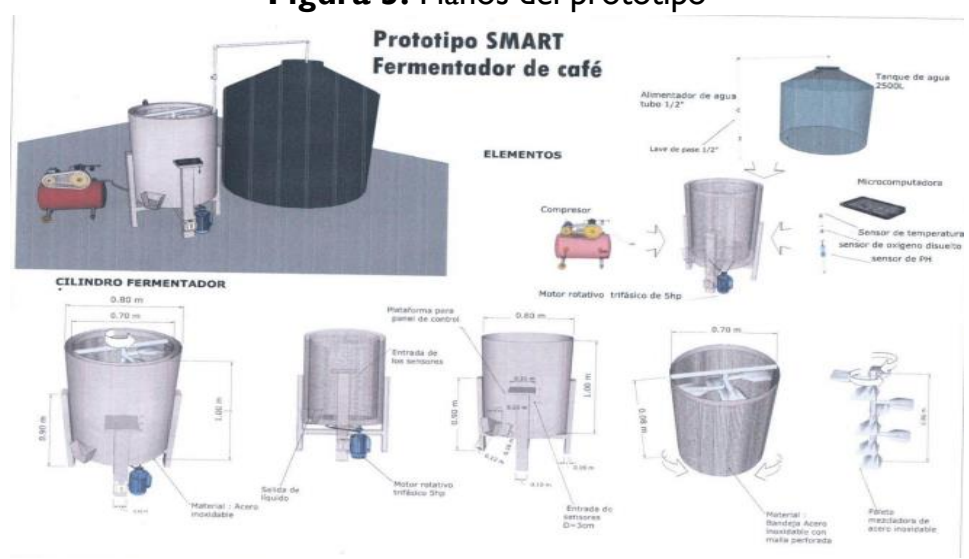

Figura 4. Planos del Prototipo

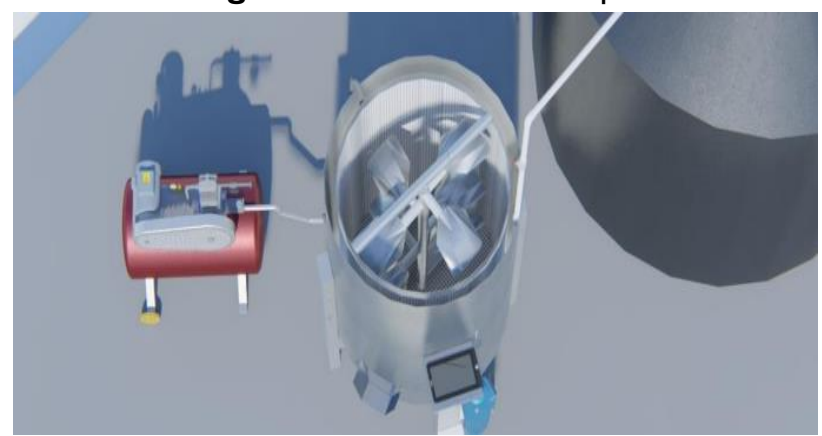

El diseño del prototipo permitió:

- El modelo cilíndrico garantiza una homogeneidad de fermentación de sustrato de café en al menos $99 \%$ respecto al modelos cuadrados puede llegar a un $90 \%$ de homogeneidad

- El modelo cilíndrico ayuda a escurrir en menos tiempo el mucilago del sustrato por su posición vertical y el sustrato apilado se presiona, generando un $30 \%$ más efectivo que modelo tipo cuadrado.

- El proceso de lavado es más versátil y utilización de menos agua por la posición y la presión que genera por la gravedad.

- El sistema de sensores se adapta para ambos modelos.

- El algoritmo inteligente sería más provechoso con el modelo cilíndrico.

\section{Ensamblaje del prototipo fermentador de café}

\section{Mecánica-eléctrica}

El proceso de ensamblaje se inició de acuerdo al modelo de diseño elegido por el cual se inició a partir de ahí y realizar el prototipo, el cual se basó de bandeja redonda con dimensiones de 0.80 centímetros de diámetro con altura de 100 centímetros.

- Base de la estructura. Se lograron realizar el rolado de la parte base del cilindro, el cual fue con planchas de acero inoxidable de $2 \mathrm{~mm}$ de espesor, el cual se lograron realizar 
rolar en las dimensiones antes descritas de 100 centímetros de altura y 0,80 centímetros de diámetro.

Figura 5. Base ensamblada del Prototipo

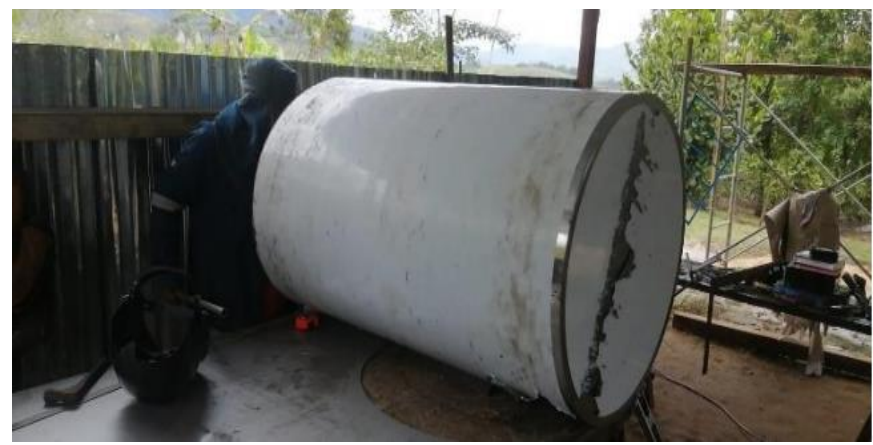

Seguidamente se logró adherir sistema de tapas y patas (sostener su estructura).

Figura 6. Base ensamblada del prototipo y su tapa.

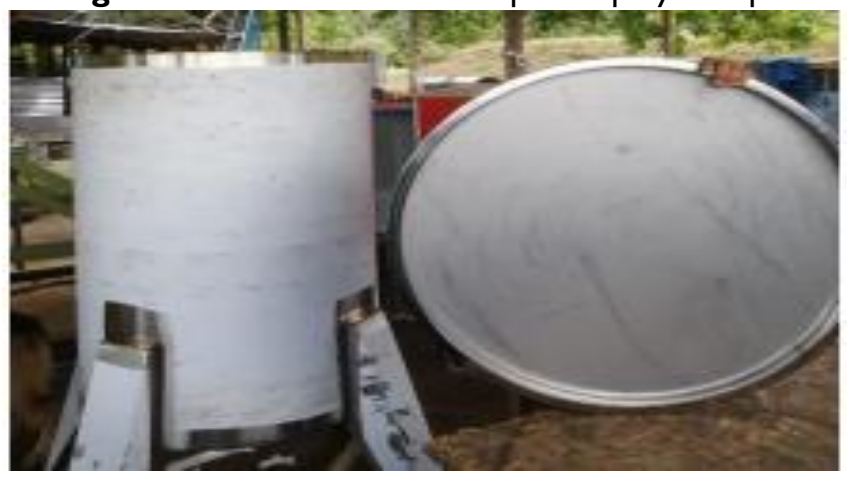

Sistema interno de paletas. Se hizo un eje principal de I I/5 el diámetro de tubo redondo de acero inoxidable, esto está conectado a dos chumaceras y de ahí a un motoreductor de 2 HP. Paralelamente se adhirió planchas cortadas de acero inoxidable en forma de remo para generar movimiento de uniformidad en la masa.

Figura 7. Sistema interno de paletas por dentro.

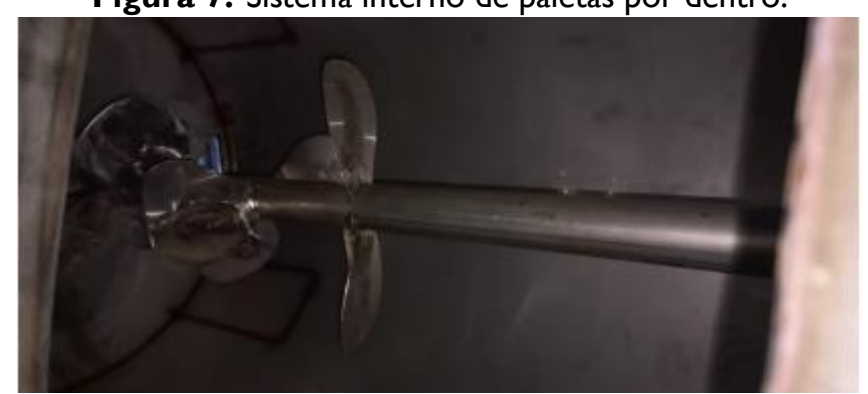

Sistema de entradas y salidas. El sistema de entrada del grano es por la parte de la tapa, que se realizó un ajuste de diseño final, el cual se hizo una compuerta para el ingreso y su sellado hermético para garantizar las dos fases: Anaeróbico y aeróbico. Para la salida del CO2 acumulado se hizo una salida por la tapa con una manguera hacia un sistema de acuoso para evitar el ingreso de bacterias ajenas y oxígeno. 
Figura 8. Sistema de entradas y salidas de grano.

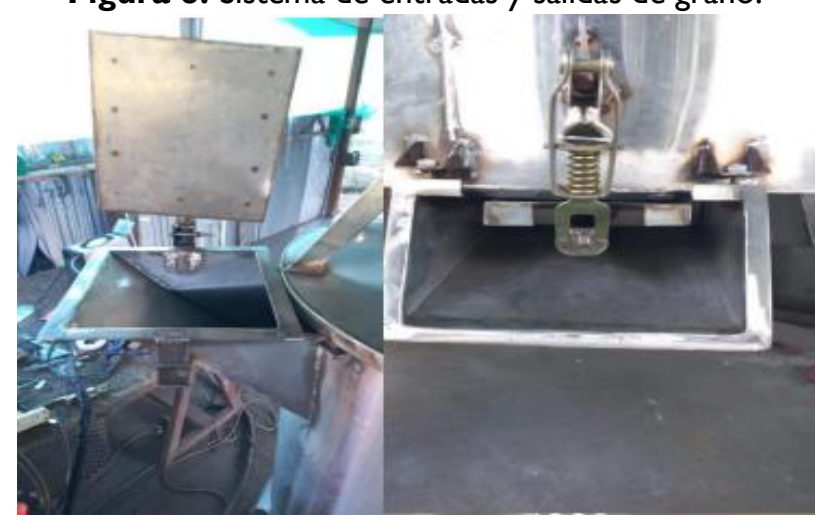

Sistema de alimentación eléctrica. Se procedió a instalar sistema de inyección eléctrico, tomando en cuenta que el local de la cooperativa cuenta con una potencia activa destinada de $10 \mathrm{KW}$ en trifásica de parte del proveedor de generación (Electro oriente). Se procedió a poner sistema de cuchillas y contactores:

Motor Cuchilla térmica de 100 amperios y un contactor de igual proporción de potencia. Sistema de válvulas de inyección de agua Cuchilla térmica de 40 amperios y un contactor de igual proporción de potencia. Modulo electrónico. Cuchilla térmica de 40 amperios y un contactor de igual proporción de potencia.

\section{Ensamblaje Electrónico}

El sistema electrónico del prototipo se dividió en dos fases:

- Control Dentro del sistema de control se realizó una conexión al sensor de temperatura de masa y se conectó a una placa con microprocesador ATmega640 del cual se procedió a codificarle para darle rangos para el control respectivo.

Figura 9. Flujograma del proceso electrónico de control.

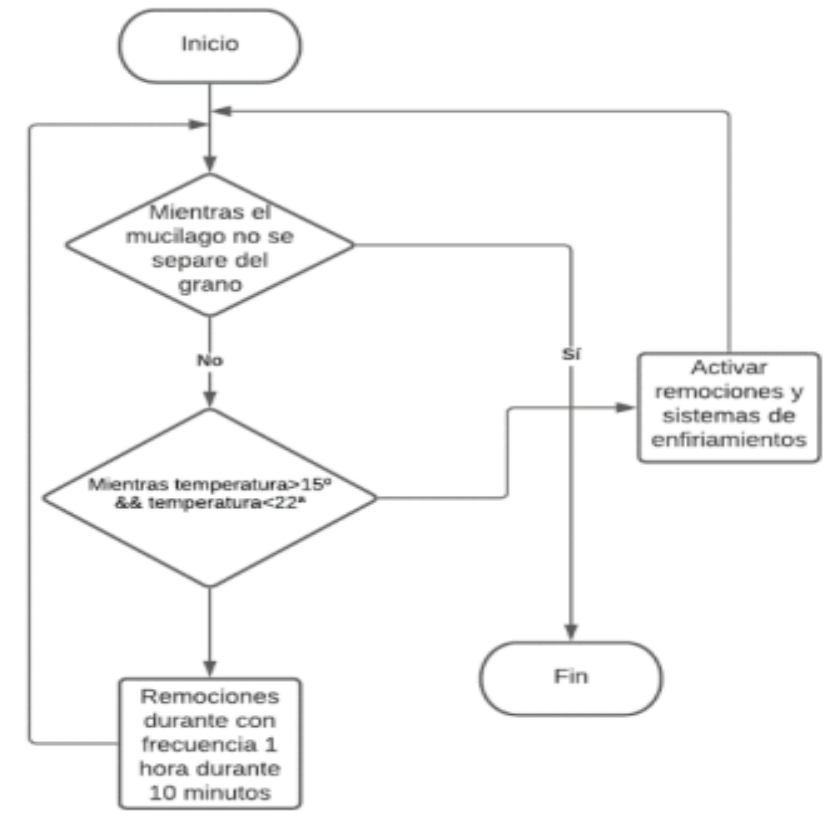


Monitoreo (captura y guardado de datos). El monitoreo de los datos se realizó instalando sensores conectados a la placa ATmega640 y a la conexión de microcomputadora para su guardado final, y se pueda descargar los datos para su análisis respectivo.

Figura 10. Sistema electrónico

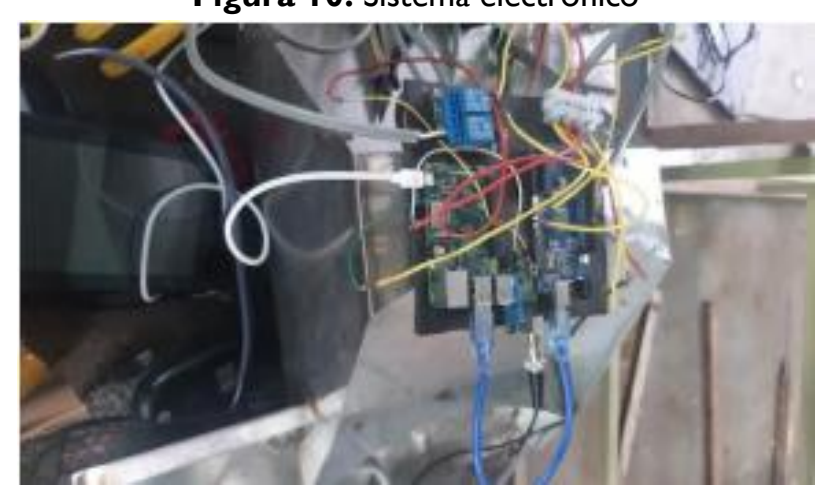

Figura I I. Sistema de monitoreo en construcción operando

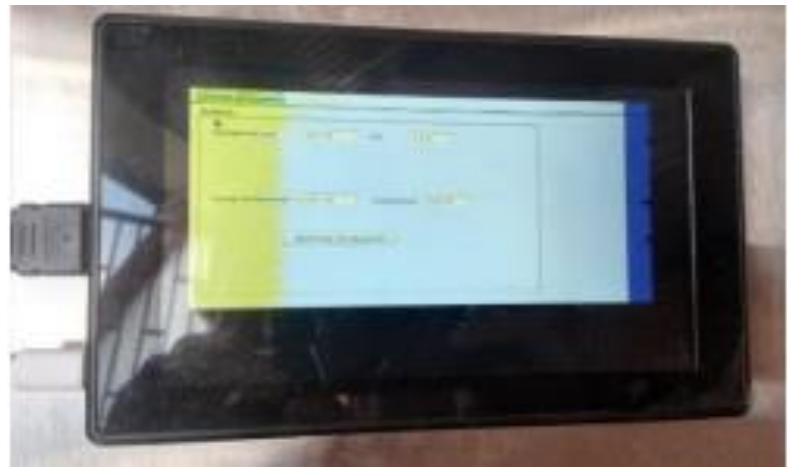

Las conexiones de la placa ATM256 con la microcomputadora se hicieron mediante protocolo MODBUS por puerto serial. La programación de bajo nivel fue desarrollada en C++, la captura y guardado de datos en JAVA y Gestor de base de datos MYSQL.

Software. El proceso de desarrollo se hizo en dos fases:

- Programación de la placa ATM256(control) La placa se codificó en C++ el cual a través de librerías se conectó a los sensores de $\mathrm{pH}$ y temperatura. La misma placa se generó el algoritmo de control poniendo los parámetros que se necesitan.

- Codificación del software monitoreo El software se elaboró con el lenguaje de programación java, con multihilo continuo para conectarse mediante serial a la placa ATM256. La estructura de archivos fue la siguiente:

Figura I2. Estructura de archivos

5 lib
5 model
6 orm
6 view


Donde: lib contiene las librerías de conexiones seriales y base de datos. model contiene los archivos para manejador de datos y conexión ORM. orm contiene los archivos ORM para conexión a la base de datos. View son los formularios desktops del programa.

En la base de datos un registro de evaluaciones se guarda cada vez que se inicia un batch de evaluación, y otro registro de cada 30 segundos.

Figura 13. Pantalla inicial donde se dan las opciones de generar una evaluación y exportar los datos.

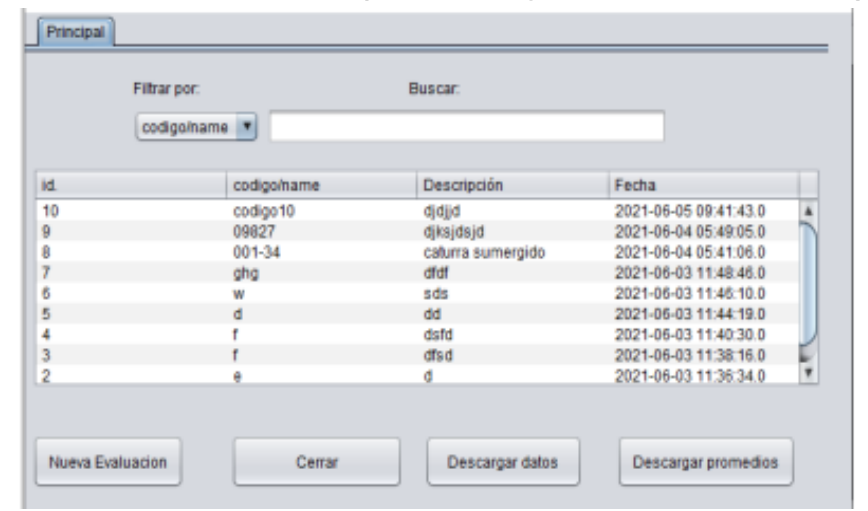

Figura 14. Pantalla del registro de una evaluación.

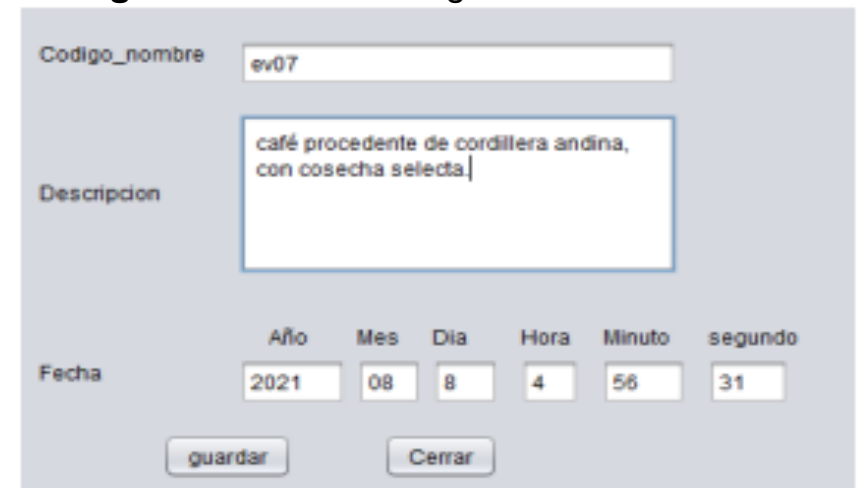

Figura 15. Pantalla de muestra del monitoreo en tiempo real.

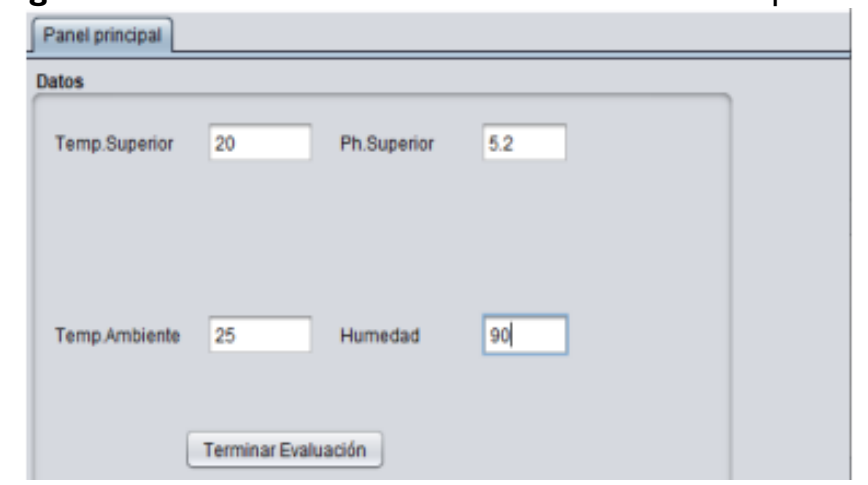

\section{Estructura de la validación del prototipo fermentador.}

\section{Operacionalidad de las variables}

- Variable independiente: Prototipo fermentador inteligente con tecnología de fermentación controlado. 
Tabla I. Indicadores de la variable independiente

\begin{tabular}{|c|c|c|c|c|}
\hline İtem & Indicador & Dimensión & Escala de medición & Instrumento \\
\hline $\mathbf{I}$ & $\begin{array}{c}\text { Resultados sensoriales } \\
\text { final del grano procesado } \\
\text { por el prototipo }\end{array}$ & Catación de las muestras & $\begin{array}{c}\text { Puntos según formato } \\
\text { SCAA }\end{array}$ & $\begin{array}{c}\text { Fichas de recolección } \\
\text { con formatos SCAA }\end{array}$ \\
\hline $\mathbf{2}$ & $\begin{array}{c}\text { Medición de temperatura } \\
\text { en tiempo real }\end{array}$ & $\begin{array}{c}\text { Temperatura de la masa del } \\
\text { grano de café durante el } \\
\text { proceso de fermentación }\end{array}$ & $\begin{array}{c}\text { Grados centígrados/30 } \\
\text { segundos }\end{array}$ & Base de datos \\
\hline $\mathbf{3}$ & $\begin{array}{c}\text { Medición de pH en tiempo masa de grano de } \\
\text { real durante el proceso de } \\
\text { fermentación }\end{array}$ & $\begin{array}{c}\text { Grados centígrados/30 } \\
\text { segundos }\end{array}$ & Base de datos \\
\hline
\end{tabular}

Tabla 2. Evaluaciones realizadas a las pruebas con el prototipo.

\begin{tabular}{|c|c|c|c|c|}
\hline $\begin{array}{c}\text { Código } \\
\text { Evaluación }\end{array}$ & Origen del grano & Repetición & $\begin{array}{l}\text { Puntaje } \\
\text { SCAA }\end{array}$ & Condición \\
\hline EVAOI & \multirow{3}{*}{$\begin{array}{c}\text { Zona baja (I I } 30 \text { msnm), Nuevo Edén, } \\
\text { productor Sr. Jeremías Aponte } \\
\text { Jiménez. }\end{array}$} & Primera & 82.5 & Anaeróbico seco. \\
\hline EVA02 & & Primera & 83.7 & Anaeróbico $30 \%$. \\
\hline EVA03 & & Primera & 83.00 & Anaeróbico 50\%. \\
\hline EVA04 & \multirow{3}{*}{$\begin{array}{c}\text { Zona alta (I600 msnm), Alto Carrizal, } \\
\text { productor Abilio Sayago Sóndor. }\end{array}$} & Segunda & 84.5 & Anaeróbico seco. \\
\hline EVA05 & & Segunda & 85.00 & Anaeróbico $30 \%$. \\
\hline EVA06 & & Segunda & 84.7 & Anaeróbico 50\%. \\
\hline
\end{tabular}

\section{Primera Repetición}

\section{Evaluación EVAOI}

Se realizaron el análisis de calidad física y organoléptica de 40 muestras de café Honey; los resultados se presentan en la siguiente tabla.

Tabla 3. Evaluación de la fermentación anaeróbico solido (sin agua)

\begin{tabular}{|c|c|c|c|c|c|c|c|c|c|c|c|c|c|c|c|c|c|c|c|}
\hline & $\mathbf{0}$ & $\mathbf{I}$ & $\mathbf{2}$ & $\mathbf{3}$ & $\mathbf{4}$ & $\mathbf{5}$ & $\mathbf{6}$ & $\mathbf{1 0}$ & $\mathbf{1 3}$ & $\mathbf{1 6}$ & $\mathbf{1 7}$ & $\mathbf{1 8}$ & $\mathbf{1 9}$ & $\mathbf{2 0}$ & $\mathbf{2 1}$ & $\mathbf{2 2}$ & $\mathbf{2 3}$ & $\mathbf{2 4}$ & $\mathbf{2 5}$ \\
\hline${ }^{\circ}$ Brix & 10.1 & 11.0 & 11.0 & 11.1 & 11.3 & 11.9 & 12 & 12 & 12 & 12 & 12.3 & 12.5 & 12 & 11.5 & 11.5 & 11.5 & 11.5 & 11.5 & 11.5 \\
\hline $\mathbf{~ p H}$ & 5.40 & 5.37 & 5.34 & 5.30 & 5.27 & 5.23 & 5.21 & 5.18 & 5.16 & 5.15 & 5.03 & 4.93 & 4.69 & 4.47 & 4.34 & 4.08 & 3.86 & 3.51 & 3.3 \\
\hline $\mathbf{T}^{\circ}$ masa ( $\left.{ }^{\circ} \mathbf{C}\right)$ & 22.6 & 20.6 & 20.5 & 19.9 & 19.1 & 18.8 & 18.1 & 16.6 & 17.9 & 18.6 & 21 & 21.5 & 22 & 27.8 & 26.8 & 30 & 28.8 & 28.4 & 24 \\
\hline $\mathbf{T}^{\circ}$ ambiente ( $\left.{ }^{\circ} \mathbf{C}\right)$ & 23.3 & 20.3 & 20.1 & 19.1 & 18 & 17.3 & 16 & 15.8 & 17.3 & 18.8 & 20.1 & 21 & 23 & 29 & 26 & 28 & 28 & 23 & 22 \\
\hline
\end{tabular}

Las temperaturas presentaron variaciones en masa y ambiente; ambos factores presentan similitudes en comportamiento durante el proceso siendo estadísticamente iguales, decreciente en la noche y madrugada y aumenta con el paso de las horas de la tarde. Con relación a los grados Brix, se tiene una lectura inicial de $10.1^{\circ} \mathrm{Bx}$ y estos aumentan gradualmente, debido a la sedimentación o acumulación de mucílago desprendido en el balde durante las 18 primeras horas, luego las lecturas se tornan decrecientes hasta la hora 20 y se mantuvo estático hasta su interrupción (con una Lf: I I. $5^{\circ} \mathrm{Bx}$ ). El pH del café fresco en baba es ácido al inicio (teniendo una Li: de 5.40) disminuye durante toda la fermentación debido a la disociación de ácidos y temperaturas de masa y ambiente, principalmente el ácido láctico y el aumento de la temperatura ambiental y de masa. Esta se vio interrumpida a un pH de 3.3 y duró 24 horas con 40 minutos. 


\section{Evaluación EVA02}

Tabla 4. Evaluación de la fermentación anaeróbico (al 30\% sumergido en agua).

\begin{tabular}{|c|c|c|c|c|c|c|c|c|c|c|c|c|c|c|c|c|c|}
\hline & 0 & I & 2 & 3 & 4 & 5 & 6 & 10 & 13 & 16 & 17 & 18 & 19 & 20 & 21 & 22 & 23 \\
\hline & $\begin{array}{l}\frac{\sum}{n} \\
\frac{n}{\ddot{r}}\end{array}$ & $\frac{\sum}{\frac{\Sigma}{n}}$ & $\frac{\sum}{\frac{\Sigma}{n}}$ & $\begin{array}{l}\frac{\sum}{0} \\
\underline{n} \\
\end{array}$ & $\begin{array}{l}\frac{\Sigma}{\alpha} \\
\frac{\omega}{\ddot{\infty}}\end{array}$ & $\begin{array}{l}\frac{\sum}{n} \\
\frac{n}{\ddot{a}}\end{array}$ & $\begin{array}{l}\frac{\Sigma}{0} \\
\underline{\underline{n}} \\
\underline{\underline{0}}\end{array}$ & $\begin{array}{l}\sum_{\alpha} \\
\frac{\tilde{\alpha}}{\ddot{N}}\end{array}$ & $\begin{array}{l}\sum_{\alpha} \\
\frac{n}{\ddot{n}}\end{array}$ & $\begin{array}{l}\frac{\sum}{<} \\
\frac{n}{\infty}\end{array}$ & $\begin{array}{l}\frac{\sum}{\alpha} \\
\frac{n}{\ddot{a}}\end{array}$ & $\begin{array}{l}\frac{\sum}{\alpha} \\
\stackrel{n}{0} \\
\underline{0}\end{array}$ & $\begin{array}{l}\Sigma \\
\Sigma \\
ㅡ \\
\end{array}$ & $\begin{array}{l}\Sigma \\
\underline{\Sigma} \\
\underline{\underline{n}} \\
\underline{\underline{I}}\end{array}$ & 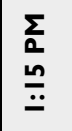 & $\begin{array}{l}\frac{\sum}{a} \\
\underline{n} \\
\ddot{n}\end{array}$ & $\begin{array}{l}\frac{\sum}{\alpha} \\
\frac{0}{\ddot{m}}\end{array}$ \\
\hline${ }^{\circ}$ Brix & 9.5 & 9.5 & 10 & 10.5 & 10.8 & 11.5 & 11.5 & 12 & 12 & 12 & 12.5 & 12.5 & 12 & 12 & 11.5 & 11.3 & 10.5 \\
\hline $\mathrm{pH}$ & 5.91 & 5.83 & 5.75 & 5.68 & 5.6 & 5.51 & 5.46 & 5.38 & 5.29 & 5.11 & 5.03 & 4.7 & 4.21 & 3.99 & 3.76 & 3.45 & 3.20 \\
\hline $\mathbf{T}^{\circ} \operatorname{masa}\left({ }^{\circ} \mathrm{C}\right)$ & 22 & 22.5 & 20.9 & 20 & 20.1 & 18.2 & 18 & 17.3 & 18.6 & 18 & 20.1 & 21.7 & 22 & 27.2 & 27.1 & 25 & 26.9 \\
\hline $\mathrm{T}^{\circ}$ amb. $\left({ }^{\circ} \mathrm{C}\right)$ & 23.3 & 20.3 & 20.1 & 19.1 & 18 & 17.3 & 16 & 15.8 & 17.3 & 18.8 & 20.1 & 21 & 23 & 29 & 26 & 28 & 28 \\
\hline
\end{tabular}

Las temperaturas presentaron variaciones de masa y ambiente $y$ ambos factores presentan similitudes durante la fermentación, siendo estadísticamente iguales, con una forma decreciente en horas de la noche y madrugada y aumenta con el transcurrir de las horas de la tarde.

Con relación a los grados Brix, se tiene una lectura inicial de $9.5^{\circ} \mathrm{Bx}$ y estos aumentan gradualmente a medida que transcurre el tiempo, debido a la sedimentación o acumulación de mucílago desprendido en el balde durante las 18 primeras horas, pero luego las lecturas se tornan decrecientes hasta su interrupción (con una Lf: $10.5^{\circ} \mathrm{Bx}$ ).

El pH del café fresco en baba (mucílago) es ácido al inicio (teniendo una Li: de 5.9l) disminuye gradualmente durante toda la fermentación debido a la disociación de ácidos y temperaturas de masa y ambiente, principalmente el ácido láctico y el aumento de la temperatura ambiental y de la masa. La interrupción de esta la fermentación fue con un $\mathrm{pH}$ de 3.2 y duró 22 horas con 55 minutos.

\section{Evaluación EVA03}

Tabla 5. Evaluación de la fermentación anaeróbico (al 50\% sumergido en agua).

\begin{tabular}{|c|c|c|c|c|c|c|c|c|c|c|c|c|c|c|c|c|c|c|c|}
\hline & 0 & I & 2 & 3 & 4 & 5 & 6 & 10 & 13 & 16 & 17 & 18 & 19 & 20 & 21 & 22 & 23 & 24 & 25 \\
\hline & $\begin{array}{l}\frac{\sum}{\alpha} \\
\frac{\operatorname{Ln}}{\dot{\sigma}}\end{array}$ & $\begin{array}{l}\frac{\Sigma}{n} \\
\frac{\omega}{\alpha}\end{array}$ & $\begin{array}{l}\frac{\Sigma}{a} \\
\underline{\underline{n}} \\
\underline{\underline{0}}\end{array}$ & $\begin{array}{l}\frac{\sum}{n} \\
\frac{\operatorname{m}}{\ddot{n}}\end{array}$ & $\begin{array}{l}\frac{\Sigma}{a} \\
\frac{\omega}{\ddot{0}}\end{array}$ & $\begin{array}{l}\frac{\Sigma}{a} \\
\frac{n}{\pi}\end{array}$ & $\begin{array}{l}\frac{\Sigma}{n} \\
\frac{\operatorname{n}}{\ddot{\infty}}\end{array}$ & 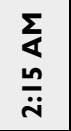 & $\begin{array}{l}\frac{\sum}{<} \\
\frac{n}{\ddot{n}}\end{array}$ & $\begin{array}{l}\frac{\sum}{\alpha} \\
\frac{n}{\infty}\end{array}$ & $\frac{\sum}{\frac{\sum}{\alpha}}$ & 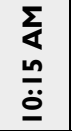 & $\begin{array}{l}\Sigma \\
\underline{\Sigma} \\
\underline{\underline{n}} \\
\underline{\underline{\underline{n}}}\end{array}$ & $\begin{array}{l}\Sigma \\
\underline{\Sigma} \\
\underline{\underline{\Lambda}} \\
\underline{\underline{I}}\end{array}$ & $\begin{array}{l}\frac{\Sigma}{0} \\
\underline{\underline{n}} \\
\ddot{-}\end{array}$ & $\begin{array}{l}\frac{\Sigma}{n} \\
\underline{\dot{n}} \\
\dot{i}\end{array}$ & $\begin{array}{l}\sum_{n} \\
\frac{\operatorname{Ln}}{\ddot{m}}\end{array}$ & $\begin{array}{l}\frac{\Sigma}{n} \\
\underline{n} \\
\dot{\dot{r}}\end{array}$ & $\begin{array}{l}\frac{\Sigma}{n} \\
\frac{\operatorname{m}}{\ddot{n}}\end{array}$ \\
\hline${ }^{\circ}$ Brix & 8.5 & 8.5 & 8.5 & 9.1 & 9.1 & 9.5 & 9.5 & 10.3 & 10 & 10 & 9.8 & 9.5 & 9 & 9 & 9 & 9 & 9 & 9 & 9 \\
\hline pH & 5.71 & 5.65 & 5.61 & 5.58 & 5.55 & 5.5 & 5.45 & 5.38 & 5.31 & 5.25 & 5.14 & 5.08 & 4.84 & 4.64 & 4.24 & 3.98 & 3.83 & 3.45 & 3.1 \\
\hline $\mathbf{T}^{\circ}$ masa $\left({ }^{\circ} \mathrm{C}\right)$ & 22.3 & 20.9 & 20.7 & 20.5 & 20.0 & 19.6 & 18.1 & 16.4 & 19.5 & 19.7 & 21.0 & 21.7 & 23.0 & 25.5 & 25.5 & 28 & 29 & 28.4 & 25.5 \\
\hline & 23.3 & 20.3 & 20.1 & 19.1 & 18 & 17.3 & 16 & 15.8 & 17.3 & 18.8 & 20.1 & 21 & 23 & 29 & 26 & 28 & 28 & 23 & 22 \\
\hline
\end{tabular}

La temperatura presentó variaciones de masa y ambiente, su comportamiento tiene similitud con la fermentación anaeróbico sólido y anaeróbico sumergido al $30 \%$ en agua; es decir, con tendencia primero decreciente, luego creciente y por último siendo estadísticamente iguales.

Con relación a los grados Brix, se tiene una lectura inicial de $8.5^{\circ} \mathrm{Bx}$, aumentan lentamente debido a la disolución de sustancias en agua durante las 10 primeras horas, luego las lecturas se tornan decrecientes hasta las 18 horas, a partir de las 19 horas de fermentación, los ${ }^{\circ} \mathrm{Bx}$ se tornan estáticos hasta el momento de su interrupción (con una Lf: 9 $\mathrm{Bx}$ ). 
El pH del café fresco en baba (mucílago) es ácido al inicio (teniendo una Li: de 5.7I) y disminuye durante todo el proceso de fermentación debido a la acumulación de agua, poca presencia de oxígeno y la disociación de ácidos es lenta pero progresiva, principalmente el ácido alcohólico y láctico. La interrupción de esta fermentación fue con un $\mathrm{pH}$ de 3.1 y duró 25 horas.

Para todos, existe tendencia creciente de ${ }^{\circ} \mathrm{Bx}$ con el transcurrir de las horas y solo para la fermentación anaeróbica sumergido al $50 \%$ en agua es la que menos ${ }^{\circ} \mathrm{Bx}$ tuvo en esta práctica, esto se debe a la cantidad de agua colocada en el balde, pudo haber sido que se colocó una proporción más de lo estimado, haciendo que la lectura inicial sea baja a diferencia de los 3 tipos de fermentación restantes y que a las 19 horas se mantiene estático las lecturas en $9{ }^{\circ} \mathrm{Bx}$, estas 3 últimas fermentaciones (anaeróbico sumergido al $30 \%$ en agua, aeróbico y anaeróbico sólido) la acumulación de azúcares crece al iniciar la fermentación y luego decrece a las 18 horas sin estabilidad alguna hasta su interrupción para el proceso de lavado a excepción de la fermentación Anaeróbico sólido que decreció hasta mantener una estática de $11.5^{\circ} \mathrm{Bx}$ hasta su interrupción.

\section{Segunda Repetición}

\section{Evaluación EVA04}

Tabla 6. Evaluación de la fermentación anaeróbico sólido.

\begin{tabular}{|c|c|c|c|c|c|c|c|c|c|c|c|c|c|c|c|c|c|c|c|c|c|c|c|}
\hline & 0 & I & 15 & 16 & 17 & 18 & 19 & 20 & 21 & 22 & 23 & 24 & 25 & 39 & 40 & 41 & 42 & 43 & 44 & 45 & 46 & 47 & 48 \\
\hline & 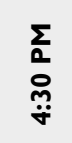 & 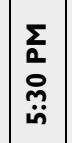 & $\begin{array}{l}\Sigma \\
\Sigma \\
\stackrel{\Sigma}{0} \\
\stackrel{m}{\sim}\end{array}$ & 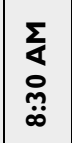 & 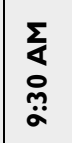 & 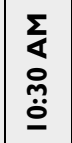 & $\begin{array}{l}\underline{\Sigma} \\
\stackrel{\alpha}{o} \\
\stackrel{\rho}{\underline{m}} \\
\underline{=}\end{array}$ & 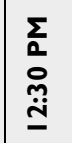 & $\begin{array}{l}\boldsymbol{\Sigma} \\
\mathbf{n} \\
\stackrel{\rho}{\underline{m}}\end{array}$ & $\begin{array}{l}\sum_{a} \\
\dot{\infty} \\
\dot{\sim}\end{array}$ & $\begin{array}{l}\sum_{a} \\
\text { o } \\
\dot{m}\end{array}$ & 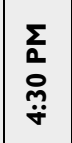 & 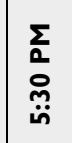 & 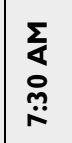 & 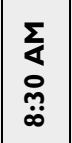 & 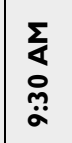 & 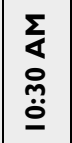 & $\begin{array}{l}\Sigma \\
\Sigma \\
o \\
\stackrel{m}{\underline{m}} \\
\underline{=}\end{array}$ & 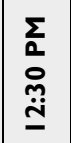 & 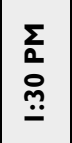 & $\begin{array}{l}\sum_{0} \\
0 \\
\stackrel{M}{N}\end{array}$ & 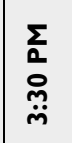 & $\begin{array}{l}\Sigma \\
\Sigma \\
0 \\
\stackrel{\Sigma}{0} \\
\dot{+}\end{array}$ \\
\hline${ }^{\circ}$ Brix & 13.5 & 13.5 & 14.9 & 14.5 & 14.5 & 14.3 & 14.3 & 14.3 & 14.3 & 14.3 & 14.3 & 14.3 & 14 & 13.8 & 13.8 & 13.8 & 13.3 & 12.9 & 12.9 & 12.9 & 12.5 & 12.5 & 12.5 \\
\hline $\mathrm{pH}$ & 4.42 & 4.40 & 4.38 & 4.36 & 4.31 & 4.30 & 4.30 & 4.29 & 4.26 & 4.26 & 4.26 & 4.26 & 4.25 & 4.17 & 4.15 & 4.14 & 4.14 & 4.13 & 4.08 & 3.89 & 3.58 & 3.33 & 3.11 \\
\hline $\begin{array}{c}\mathbf{T}^{\circ} \text { masa } \\
\left({ }^{\circ} \mathrm{C}\right)\end{array}$ & 23.4 & 22.3 & 16.9 & 18 & 18 & 19.6 & 21 & 22 & 23.6 & 23.8 & 23.4 & 22.7 & 21 & 16.3 & 16.9 & 17.4 & 19.7 & 21 & 22.1 & 22.7 & 23 & 23.9 & 23 \\
\hline $\begin{array}{c}T^{\circ} \mathbf{a m b} \\
\left({ }^{\circ} \mathrm{C}\right)\end{array}$ & 24.3 & 20.3 & 20.1 & 23.3 & 25.9 & 27.7 & 28.1 & 27.7 & 27.7 & 27.6 & 22.4 & 20.5 & 18.6 & 19.2 & 20.1 & 22.3 & 26.7 & 26.9 & 26.3 & 23.9 & 28 & 29.4 & 27 \\
\hline
\end{tabular}

La temperatura presentó variaciones tanto de masa y ambiente y se manifiesta de forma decreciente hasta la hora 15 y la hora 39, luego aumenta conforme está pasando el tiempo desde las 16 y $4 \mathrm{I}$ horas, llegando aumentar más de $29^{\circ} \mathrm{C}$, pudiendo acelerar la fermentación.

Con relación a los grados Brix en cerezo, lectura promedio $17.5^{\circ} \mathrm{Bx}$ inicial I3.5 en masa fermentada que aumenta debido a la sedimentación o acumulación de mucílago desprendido en el balde durante las 15 horas, luego las lecturas van decreciendo hasta las 45 horas y por último, se tornan estáticas desde las 46 hasta su interrupción, a medida que transcurre el tiempo disminuye los ${ }^{\circ} \mathrm{Bx}$ hasta su última hora con una Lf: $12.5^{\circ} \mathrm{Bx}$.

El pH del café fresco en baba (mucílago) es ácido al inicio (teniendo una Li: 4.42), disminuye lentamente durante todo el proceso de fermentación debido al piso altitudinal y en relación al comportamiento de la $\mathrm{T}^{\circ}$ de la masa, la interrupción a esta fermentación se dio a un $\mathrm{pH}$ de $3 . \mathrm{I}$ y está fermentación duró 47 horas con 30 minutos. 


\section{Evaluación EVA05}

Tabla 7: Evaluación de la fermentación anaeróbico (al $50 \%$ sumergido en agua)

\begin{tabular}{|c|c|c|c|c|c|c|c|c|c|c|c|c|c|c|c|c|c|c|c|c|c|c|c|}
\hline & 0 & I & 15 & 16 & 17 & 18 & 19 & 20 & 21 & 22 & 23 & 24 & 25 & 39 & 40 & 41 & 42 & 43 & 44 & 45 & 46 & 47 & 48 \\
\hline & 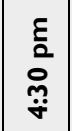 & $\begin{array}{l}\text { छे } \\
\text { 윰 } \\
\text { in }\end{array}$ & 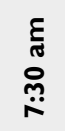 & $\begin{array}{l}\text { ह్స } \\
\text { ஓे } \\
\text { ले }\end{array}$ & 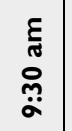 & $\begin{array}{l}\varepsilon \\
\text { ह } \\
\text { o } \\
\text { ஸे }\end{array}$ & 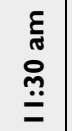 & 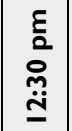 & $\begin{array}{l}\stackrel{\varepsilon}{g} \\
\stackrel{\rho}{\stackrel{m}{-}}\end{array}$ & 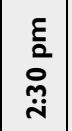 & $\begin{array}{l}\xi \\
\stackrel{\xi}{0} \\
\stackrel{\rho}{m}\end{array}$ & 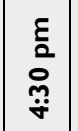 & 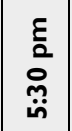 & 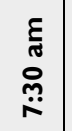 & $\begin{array}{l}\text { है } \\
\text { ळे } \\
\text { ले } \\
\ddot{0}\end{array}$ & 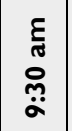 & $\begin{array}{l}\xi \\
\text { है } \\
\text { ભ } \\
\text { ஸ் }\end{array}$ & 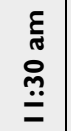 & 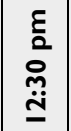 & 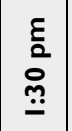 & $\begin{array}{l}\xi \\
\text { हे } \\
\text { ભ̀ } \\
\dot{\sim}\end{array}$ & $\begin{array}{l}\xi \\
\text { gे } \\
\stackrel{m}{m} \\
\ddot{m}\end{array}$ & 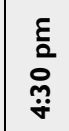 \\
\hline${ }^{\circ}$ Brix & 8.3 & 8.3 & 12 & 12.5 & 12.5 & 12.5 & 13 & 13 & 13 & 12.5 & 12.5 & 12.5 & 12.5 & 12 & 12 & 12 & 11.8 & 11.3 & II.I & 11.1 & II & II & II \\
\hline pH & 5.39 & 5.32 & 5.18 & 5.16 & 5.13 & 4.93 & 4.72 & 4.52 & 4.33 & 4.31 & 4.29 & 4.29 & 4.27 & 4.18 & 4.18 & 4.15 & 4.15 & 4.13 & 3.93 & 3.87 & 3.59 & 3.35 & 3.10 \\
\hline $\mathrm{T}^{\circ} \operatorname{masa}\left({ }^{\circ} \mathrm{C}\right)$ & 23.3 & 22 & 17.1 & 17.2 & 18 & 19.4 & 21 & 21.7 & 23.1 & 23.3 & 23.1 & 22.6 & 20.3 & 16 & 16.6 & 17.5 & 19.5 & 20.9 & 21.9 & 22.4 & 22.7 & 23.3 & 23 \\
\hline $\begin{array}{c}\mathbf{T}^{\circ} \text { ambiente } \\
\left({ }^{\circ} \mathrm{C}\right)\end{array}$ & 24.3 & 20.3 & 20.1 & 23.3 & 25.9 & 27.7 & 28.1 & 27.7 & 27.7 & 27.6 & 22.4 & 20.5 & 18.6 & 19.2 & 20.1 & 22.3 & 26.7 & 26.9 & 26.3 & 23.9 & 28 & 29.4 & 25. \\
\hline
\end{tabular}

Las temperaturas durante esta fermentación presentaron variaciones tanto de masa y ambiente, además, presentan similitudes durante el proceso de fermentación, forma decreciente en horas de la noche y madrugada y luego aumenta con el transcurrir de las horas de la tarde.

Con relación a los grados Brix en cerezo, lectura promedio $17.5^{\circ} \mathrm{Bx}$ y lectura inicial 8.3 ${ }^{\circ} \mathrm{Bx}$ en masa fermentada, estos aumentan debido a sedimentación de mucílago en balde durante las 21 primeras horas, luego las lecturas se tornan decrecientes (con una Lf: I I ${ }^{\circ} \mathrm{Bx}$ ).

El pH del café fresco en baba es ácido al inicio (teniendo una Li: de 5.39) disminuye debido a la disociación de ácidos y temperaturas, el ácido láctico y el aumento de la temperatura ambiental y de masa. La interrupción de la fermentación fue con un $\mathrm{pH}$ de 3.1 y duró 48 horas.

\section{Evaluación EVA06}

Tabla 8: Evaluación de la fermentación anaeróbico (al 50\% sumergido en agua)

\begin{tabular}{|c|c|c|c|c|c|c|c|c|c|c|c|c|c|c|c|c|c|c|c|c|c|c|c|c|}
\hline & 0 & I & 15 & 16 & 17 & 18 & 19 & 20 & 21 & 22 & 23 & 24 & 25 & 39 & 40 & 41 & 42 & 43 & 44 & 45 & 46 & 47 & 48 & 49 \\
\hline & $\begin{array}{l}\xi \\
\vdots \\
\stackrel{0}{0} \\
\stackrel{m}{\dot{\gamma}}\end{array}$ & $\begin{array}{l}\varepsilon \\
\text { हू } \\
\text { लై }\end{array}$ & 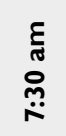 & $\begin{array}{l}\text { E } \\
\text { ○े } \\
\stackrel{m}{\infty} \\
\ddot{\infty}\end{array}$ & 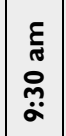 & 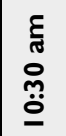 & 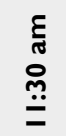 & 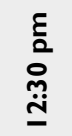 & $\begin{array}{l}\underline{\xi} \\
\text { 을 } \\
\stackrel{m}{-}\end{array}$ & 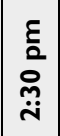 & $\begin{array}{l}\varepsilon \\
\xi \\
\circ \\
\stackrel{m}{m}\end{array}$ & 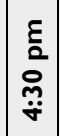 & $\begin{array}{l}\xi \\
\stackrel{\xi}{0} \\
\stackrel{m}{m} \\
\stackrel{n}{n}\end{array}$ & 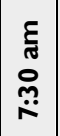 & $\begin{array}{c}\varepsilon \\
\tilde{c} \\
\stackrel{\rho}{0} \\
\ddot{\infty}\end{array}$ & 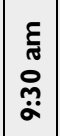 & 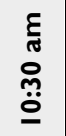 & 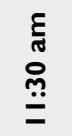 & 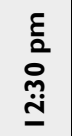 & $\begin{array}{l}\underline{\xi} \\
\stackrel{0}{0} \\
\stackrel{m}{\underline{D}}\end{array}$ & $\begin{array}{l}\varepsilon \\
\xi \\
\dot{0} \\
\stackrel{\sim}{\sim}\end{array}$ & $\begin{array}{l}\xi \\
\text { हे } \\
\stackrel{m}{m} \\
\stackrel{m}{m}\end{array}$ & $\begin{array}{c}\xi \\
\vdots \\
\circ \\
\stackrel{\oplus}{\sim}\end{array}$ & $\begin{array}{l}\xi \\
\stackrel{0}{0} \\
\stackrel{\oplus}{\tilde{*}}\end{array}$ \\
\hline${ }^{\circ}$ Brix & 7 & 7.5 & 11.5 & 11.1 & II.I & 11.1 & 11 & 11 & 10.5 & 10.5 & 10.5 & 10.5 & 10.5 & 10 & 10 & 10 & 9.6 & 9.1 & 9.1 & 9 & 9 & 9 & 8.7 & 8.5 \\
\hline $\mathrm{pH}$ & 5.54 & 5.51 & 5.31 & 5.26 & 4.93 & 4.62 & 4.42 & 4.39 & 4.36 & 4.36 & 4.36 & 4.33 & 4.33 & 4.16 & 4.16 & 4.15 & 4.15 & 4.14 & 4.11 & 5.97 & 3.84 & 3.69 & 3.45 & 3.2 \\
\hline $\mathrm{T}^{\circ} \mathrm{m}\left({ }^{\circ} \mathrm{C}\right)$ & 23.7 & 22.7 & 17.4 & 17.9 & 18.4 & 19.9 & 21 & 22.6 & 23 & 23 & 23.1 & 22.5 & 20.1 & 16.2 & 17.1 & 17.5 & 19.3 & 20.9 & 21.7 & 22.2 & 22.6 & 23 & 24.5 & 24.1 \\
\hline $\mathrm{T}^{\circ} \mathrm{amb}\left({ }^{\circ} \mathrm{C}\right)$ & 4.3 & 20.3 & 20.1 & 23.3 & 25.9 & 27.7 & 28.1 & 27.7 & 27.7 & 27.6 & 22.4 & 20.5 & 18.6 & 19.2 & 20.1 & 22.3 & 26.7 & 26.9 & 26.3 & 23.9 & 28 & 29.4 & 25.9 & 22.8 \\
\hline
\end{tabular}

\section{Conclusiones}

Se logró desarrollar un prototipo de fermentación inteligente el cual cuenta con automatización para una fermentación controlada, se obtuvieron promedios de 84 puntos SCAA de acuerdo al objetivo del proyecto, con el control de la temperatura y el monitoreo de la misma y del $\mathrm{pH}$.

En la primera repetición se incrementó la temperatura debido a factores ambientales, por el cual a la siguiente repetición se modificó el sistema de entrada de aire para inyectar mayor 
presión, pasando de inyección de $0.5 \mathrm{~m} 3 /$ minuto a $1.5 \mathrm{~m} 3 /$ minuto cuando la temperatura esta $x$ encima de 23 grados.

Los procesos anaeróbicos con sumergidos al $50 \%$ dan mejores resultados, aunque con tiempo en horas más prologados.

El proceso de fermentación con el prototipo fermentador genera una eficiencia, llegando a las metas esperadas para los clientes, con ajustes respectivos para sobrepasar los 85 puntos SCA.

El control de la temperatura ayuda a equilibrar los procesos bioquímicos los cuales se ven reflejados en las puntuaciones de tasas donde se ven que las características de sabores y fragancias son uniformes, y de perfiles suaves que le dan una característica al café por encima de 84 puntos.

\section{Referencias bibliográficas}

Al-Abdulkader, A. M., Al-Namazi, A. A., AlTurki, T. A., Al-Khuraish, M. M., \& Al-Dakhil, A. I. (2018). Optimizing coffee cultivation and its impact on economic growth and export earnings of the producing countries: The case of Saudi Arabia. Saudi Journal of Biological Sciences, 25(4), 776-782. https://doi.org/I0.1016/J.SJBS.2017.08.016

Bonilla Medina, J. (20I7). Los beneficios del consumo de café. Revista Facultad Ciencias de La Salud. Universidad Del Cauca, 19(2), 47-48.

Estevez, C. L., Bhat, M. G., \& Bray, D. B. (2017). Commodity chains, institutions, and domestic policies of organic and fair trade coffee in Bolivia. 42(3), 299-327. https://doi.org// 0.1080/2 1683565.2017.I359737

Julca-Otiniano, A., Alarcón-Águila, G., Alvarado-Huamán, L., Borjas-Ventura, R., \& CastroCepero, V. (20I8). COMPORTAMIENTO DE TRES CULTIVARES DE CAFÉ (CATIMOR, COLOMBIA Y COSTA RICA 95) EN EL VALLE DE EL PERENÉ, JUNÍN, PERÚ. CHILEAN JOURNAL OF AGRICULTURAL AND ANIMAL SCIENCES. https://scielo.conicyt.cl/pdf/chjaasc/v34n3/07/9-3890-chjaasc-00504.pdf

Junta Nacional del Café. (202I, February I). PRODUCCIÓN Y EXPORTACIONES DE CAFÉ APUNTAN A SER MEJORES DURANTE EL 2021 . https://juntadelcafe.org.pe/produccion-yexportaciones-de-cafe-apuntan-a-ser-mejores-durante-el-202I/

Lopes, A. C. A., Andrade, R. P., de Oliveira, L. C. C., Lima, L. M. Z., Santiago, W. D., de Resende, M. L. V., das Graças Cardoso, M., \& Duarte, W. F. (2020). Production and characterization of a new distillate obtained from fermentation of wet processing coffee by-products. Journal of Food Science and Technology, 57(I2), 448I-449I. https://doi.org// 0.1007/s | 3197-020-04485-4

Magalhães Júnior, A. I., de Carvalho Neto, D. P., de Melo Pereira, G. V., da Silva Vale, A., Medina, J. D. C., de Carvalho, J. C., \& Soccol, C. R. (202I). A critical techno-economic analysis of coffee processing utilizing a modern fermentation system: Implications for specialty coffee production. Food and Bioproducts Processing, I25, I4-21. https://doi.org/10.1016/j.fbp.2020.10.010 
Ocampo Lopez, O. L., \& Alvarez-Herrera, L. M. (2017). Tendencia de la producción y el consumo del café en Colombia. APUNTES DEL CENES, 36(64). https://doi.org/I0.19053/0I 203053.v36.n64.2017.5419

Pham, Y., Reardon-Smith, K., Mushtaq, S., \& Cockfield, G. (2019). The impact of climate change and variability on coffee production: a systematic review. Climatic Change, 156(4), 609630. https://doi.org//0.1007/s I0584-019-02538-y

Rojas-Ruiz, R., Alvarado-Huamán, L., Borjas-Ventura, R., Carbonell Torres, E., Castro-Cepero, V., \& Julca-Otiniano, A. (2020). Caracterización de fincas productoras de café convencional y orgánico en el valle del Alto Mayo, región San Martín, Perú. Revista de Investigación e Innovación Agropecuaria y de Recursos Naturales, 7(2), I00-II I.

Sayed, O. H., Masrahi, Y. S., Remesh, M., \& Al-Ammari, B. S. (2019). Coffee production in southern Saudi Arabian highlands: Current status and water conservation. Saudi Journal of Biological Sciences, 26(7), 1911-1914. https://doi.org//0.1016/j.sjbs.2019.03.002

Smith Dumont, E., Gassner, A., Agaba, G., Nansamba, R., \& Sinclair, F. (2019). The utility of farmer ranking of tree attributes for selecting companion trees in coffee production systems. Agroforestry Systems, 93(4), 1469-1483. https://doi.org/10.1007/s I0457-0 I80257-z

\section{Financiamiento}

Programa Nacional de Desarrollo Tecnológico e Innovación - Prolnnóvate que financió del proyecto "Desarrollo de un prototipo de secador solar tipo túnel con microclima auto controlado para obtener cafés honey de alta calidad en taza en la provincia de Moyobamba" con Contrato $\mathrm{N}^{\circ}$ 283-INNOVATEPERU-PIECI-20I9.

\section{Conflicto de intereses}

El artículo no presenta conflicto de intereses.

\section{Contribución de autores}

Carbajal-Guerreros, Ismael: Coordinador general del subproyecto, encargado de su ejecución y del logro de los objetivos. Dio visto bueno y aprobó la versión final del artículo

Pilco-Valles, Herberth: Asistente de investigación. Dio visto bueno y aprobó la versión final del artículo

García-Herrera, Flor Aracely: Asistente de investigación. Dio visto bueno y aprobó la versión final del artículo.

Coronel-Rufasto, Ivan: Análisis formal. Dio visto bueno y aprobó la versión final del artículo.

Gonzales-Diaz, Jose Rolando: Validación. Dio visto bueno y aprobó la versión final del artículo.

Cabanillas-Pardo, Lenin: Responsable del diseño técnico del dispositivo. Dio visto bueno y aprobó la versión final del artículo. 